\title{
Grid resolution study for B2-EIRENE simulation of partially detached ITER divertor plasma
}

\author{
K. Ghoos ${ }^{1}$, P. Börner ${ }^{2}$, W. Dekeyser ${ }^{1}$, A.S. Kukuskin ${ }^{3,4}$, M. \\ Baelmans $^{1}$ \\ ${ }^{1} \mathrm{KU}$ Leuven, Department of Mechanical Engineering \\ ${ }^{2}$ FZ Jülich, Institute of Energy and Climate Research (IEK-4) \\ 3 NRC "Kurchatov Institute", Moscow, Russia \\ 4 NRNU MEPhI, Moscow, Russia \\ E-mail: kristel.ghoos@kuleuven.be \\ October 2018
}

\begin{abstract}
.
This paper examines the effect of grid resolution on the solution of a B2-EIRENE simulation of a partially detached ITER divertor plasma. Due to the large amount of required computational time in coupled finite-volume/Monte-Carlo codes, simulations of the plasma edge are typically run on coarse grids. However, new averaging techniques make simulations with finer grids feasible. Starting from the original numerical grid, simulations are performed on two successively finer grids. Results of the numerical error analysis reveal that the discretization errors are large. Peak values are particularly sensitive to grid resolution and can increase more than $40 \%$ for the same model input parameters. However, this effect is partially offset by a modification of the operational space of the ITER divertor in this case. By choosing the numerical parameters more adequately, a total numerical error of only $15 \%$ has been achieved within a feasible computational time.
\end{abstract}

\section{Introduction}

Numerical simulations of the plasma edge are extremely valuable for divertor design in nuclear fusion reactors such as ITER [1]. The divertor is essential for the power and particle exhaust in fusion reactors and has to withstand large power loads. Plasma edge codes simulate plasma-neutral transport in the edge of a reactor. The worldwide used B2-EIRENE/SOLPS code [2] consists of a finite volume code (B2) that describes the plasma with fluid equations, and a Monte Carlo code (EIRENE) that uses a kinetic approach to describe the neutrals. Its latest version SOLPS-ITER [3] is adopted by the ITER Organization as the principal plasma edge simulation tool for ITER. Here, we use the SOLPS4.3 version of the code, as used in ref. [4].

Plasma edge simulations can be computationally expensive. The strong non-linear coupling between plasma and neutrals requires a very large number of iterations to reach 
a steady-state. Moreover, a large number of Monte Carlo (MC) particles per iteration is typically used to limit the statistical noise. A simulation for ITER generally requires 2 months to finish [1]. Because of the long simulation times, relatively coarse discretization grids are typically used, with only a few $10^{3}$ grid cells. However, recent developments show that an order of magnitude speed up can be obtained without losing accuracy $[5,6,7]$. By reducing the number of MC particles in each iteration and averaging the results over several iterations, it has been demonstrated that accurate solutions can be obtained in a significantly shorter computational time. Thus, it becomes feasible to use refined numerical grids and to examine the discretization error.

In addition to the discretization error, the solution has a bias and a statistical error [5]. The bias is a deterministic error, arising from the finite number of MC particles in each iteration and the non-linearities in the coupled equations. The statistical error has a probability distribution with mean 0 and standard deviation $\sigma$. The first assessment of the bias and statistical error for a partially detached ITER case (no. 2011) has been performed in ref. [6].

In this paper, we discuss a grid resolution study for the same partially detached ITER case. Additionally, a density scan is examined, where the gas puff is varied while keeping all other input variables constant. To reliably assess the discretization error, the statistical error and bias must be sufficiently small. In section 2, we describe the simulation approach and methods used to estimate all error contributions. In section 3, we discuss the grid sensitivity of the plasma heat flux and electron temperature profiles on the outer target. We also examine the peak power load, the ion saturation current and the neutral pressure for several points on the density scan. The main conclusions are summarised in section 4 .

\section{Method}

In this section, the simulation approach and the methods to estimate all error contributions are explained. In each simulation, the onset of steadiness should be determined first to decide where to start averaging. On the averaged solution there remain the statistical error, bias and discretization error. We start with estimating the statistical error. When the statistical error is made sufficiently low by averaging over many iterations, the bias can be determined by comparing two solutions on the same grid with a different number of MC particles. With a small bias, the discretization error can be assessed by comparing solutions on successively finer grids.

\subsection{Case description}

The first case examined is the B2-EIRENE ITER run (no. 2011) for a pure Deuterium plasma under partially detached conditions on the ITER F12 geometry [8]. The core particle flux is $9.1 \cdot 10^{21} \mathrm{~s}^{-1}$, the gas puff is $2.18 \cdot 10^{23} \mathrm{~s}^{-1}$ and the electron and ion input power are 19 MW each. 
Each simulation starts from flat profiles and consists of $5 \cdot 10^{5}$ outer iterations, with time step $\Delta t=9 \cdot 10^{-7}$ and relaxation factor $\tau=0.5$. Dual time stepping is used with 15 inner iterations. The EIRENE code is called with random seed initialization every outer iteration. During the simulation, averages of the variables of consecutive batches consisting of 500 (outer) iterations are stored, which is easily done on the fly. Because a significantly smaller number of MC particles per iteration can be chosen without losing accuracy on the final solution, this averaging procedure can bring a large speed up in the simulations. More details can be found in ref. [6]. It is noted that this averaging method requires steady state simulations. It should not be used for time dependent simulations or oscillating solutions [9].

To perform the density scan, the gas puff is varied, while keeping all other input parameters constant. The gas puff rates chosen are $0.59 \cdot 10^{22} \mathrm{~s}^{-1}, 0.885 \cdot 10^{22} \mathrm{~s}^{-1}$, $1.18 \cdot 10^{22} \mathrm{~s}^{-1}, 2.18 \cdot 10^{22} \mathrm{~s}^{-1}, 2.885 \cdot 10^{22} \mathrm{~s}^{-1}, 3.59 \cdot 10^{22} \mathrm{~s}^{-1}$ and $4.295 \cdot 10^{22} \mathrm{~s}^{-1}$.

\subsection{Simulation approach}

The discretization error is examined by comparing solutions on 3 grid resolutions. Each successive grid has a doubled resolution in each dimension: 74x28, 148x56, 296x112, where the first and second number indicate respectively the number of poloidal and radial grid cells. The fine grids (148x56 and 296x112) are obtained by splitting each cell of the original $74 \times 28$ grid in respectively two or four in each direction. To be able to assess all error contributions, the simulation is run six times. For each grid resolution, two simulations are executed: one with $700 \mathrm{MC}$ particles each iteration and one with 7,000 MC particles each iteration. The computational time required for the fine grid simulations can amount to a few months. An outer iteration with B2-EIRENE with 7,000 MC particles requires approximately 4 and 23 seconds for respectively the original (74x28) and the finest $(296 \times 112)$ grid on a DELL with Intel(R) XEON(R) CPU at 2.5 $\mathrm{GHz}$ using one processor only. While EIRENE needs over $90 \%$ of this time on the coarse grid, approximately $50 \%$ of the computational time is spent in B2 in simulations on the finest grid. The parallellization scheme built in EIRENE can, therefore, provide at most a factor 2 speed up in fine grid simulations.

The averaged primary plasma variables are saved in batches of 500 iterations each during the run. Dependent variables can be calculated in post-processing. This is straight-forward for variables depending on the plasma state only. The incident plasma heat flux $q_{p}$ on the target used in section 3.1 is calculated using the averaged particle and energy flux values with the following equation:

$$
q_{p}=\left(\gamma_{i, \theta} E_{p o t}+q_{e, \theta}+q_{i, \theta}\right) \theta_{1},
$$

with $\gamma_{i, \theta}$ the ion flux, $q_{e, \theta}$ the electron heat flux, $q_{i, \theta}$ the ion heat flux, $E_{\text {pot }}$ the ionization potential for Deuterium $(13.6 \mathrm{eV})$, and $\theta_{1}$ the projection angle (sine of the angle between the flux surface and the target surface). The standard deviation on this variable can be approximated through summing the variances: $\sigma_{q}^{2}=\left(\sigma_{\gamma_{i, \theta}}^{2} E_{p o t}+\sigma_{q_{e, \theta}}^{2}+\sigma_{q_{i, \theta}}^{2}\right) \theta_{1}$. 
For variables depending on the neutral state, there are two possibilities. One possibility is to run the MC code on all batches and, afterwards, take an average as the final solution. The other possibility is to perform only $1 \mathrm{MC}$ run on the averaged plasma solution. This MC run should use a large number of MC particles to achieve a low statistical error (we use 700,000 MC particles). Examples of these variables are $q_{p k}$, $p_{D T}$ and $I_{\text {sat }}$ that will be used in section 3.2 .

\subsection{Onset of steadiness}

All error estimates assume that the iterations are in a stationary regime. Iterations where a transient is present should be discarded. This number of iterations is determined by inspecting the values of the variables during the iterations [10]. When the mean of this value appears constant, it is assumed that the stationary regime is reached. For the case no. 2011, this happens after approximately 300 batches, or 150,000 iterations. Although visual determination of the end of the transient may be inaccurate, it is generally sufficient. The deterministic error due to a small remaining number of transient iterations will not influence the result strongly if the number of iterations in the stationary regime is large.

\subsection{Statistical error}

The statistical error $\epsilon_{s}$ on the overall averaged quantity is characterized by its standard deviation $\sigma$. We estimate this standard deviation based on the central limit theorem as

$$
\sigma=\frac{\sigma_{500}}{\sqrt{\frac{R}{T_{500}}}},
$$

with $\sigma_{500}$ the standard deviation of the batch values, with each batch containing the average of 500 iterations, $R$ is the number of batches over which the overall averaged value is obtained and $T_{500}$ the correlation time between the batches.

The standard deviation $\sigma_{500}$ is calculated using the sample standard deviation $s_{500}$

$$
s_{500}=\sqrt{\frac{1}{R-1} \sum_{r=1}^{R}\left(\phi_{r}-\bar{\phi}\right)^{2}},
$$

with $\phi_{r}$ the averaged value of the $r$-th batch and $\bar{\phi}$ the overall averaged value.

The correlation time $T_{500}$ is a measure for the dependency between consecutive batches and is defined as

$$
T_{500}=1+2 \sum_{\tau=1}^{\infty} \rho(\tau),
$$

with $\rho(\tau)$ the normalized autocorrelation with the lag $\tau[11]$. We use two methods to

estimate $T_{500}$ : the batching method and the autocorrelation method. With the batching method, the batch values are grouped into new batches of $M$ original batches, or $500 M$ iterations. Based on equation (2), we can write

$$
T_{500}=\frac{s_{M}^{2}}{s_{500}^{2}} M
$$


where $s_{M}$ is the sample standard deviation of the population of the batch means containing $M$ original batches of 500 iterations or $500 M$ iterations. It is assumed that the batches become independent from each other when a sufficiently large number of iterations are batched together. The specific number of iterations depends on the case specifications and the variable. To be able to estimate the sample standard deviation $s_{M}$ reliably, at least 30 batches of $500 M$ iterations should be used. This limits the value of $M$ to $\frac{R}{30}$, with $R$ the total number of original batches. For small values of the $T_{500}$, this method works well. However, when the correlation between batches is strong $\left(T_{500}>\approx 10\right), M$ cannot be chosen sufficiently large and the autocorrelation method provides a better estimate. In this method, the autocorrelation $\rho(\tau)$ is estimated for several $\tau$ and $T_{500}$ is computed based on the fitted exponential $\rho(\tau)$. More details can be found in ref. [10]. In the following examples, $T_{500}$ is calculated with both methods and the largest value is taken.

To account for the stochastic character of the statistical error and inaccuracies in the estimation of $T_{500}$, the obtained estimate for the statistical error $\sigma$ is multiplied by 3 .

\subsection{Bias}

The bias is a deterministic error originating from the finite number of the MC particles $P$ and the non-linearities in the coupled system of equations. It is defined as the expected value of the error due to a limited $P$. The fact that this error is inversely proportional to the number of MC particles $\epsilon_{b} \propto \frac{1}{P}$ for a constant grid size [5] is used to estimate the bias. Starting from two solutions, $\phi_{P}$ and $\phi_{\alpha P}$, with a different number of MC particles per iteration, $P$ and $\alpha P$ respectively, the bias reads:

$$
\epsilon_{b, P}=\alpha \frac{\phi_{P}-\phi_{\alpha P}}{1-\alpha} .
$$

In this paper, $P=7,000 \mathrm{MC}$ particles and $\alpha=0.1$. To obtain a reliable estimate of the bias, the statistical error should be sufficiently low, i.e. $\epsilon_{s, P}+\epsilon_{s, \alpha P}<<\epsilon_{b, \alpha P}-\epsilon_{b, P}$. By choosing $\alpha P$ low, the bias $\epsilon_{b, \alpha P}$ is provoked, which is beneficial to obtain a good

estimate. To take into account inaccuracies in the estimate, a safety factor of 2 is applied. The inaccuracies can come from remaining statistical errors of the solutions or from not reaching the asymptotic $\frac{1}{P}$ scaling.

Using a well-estimated bias, the estimate for the solution without bias can be constructed as $\phi_{\infty} \approx \phi_{P}+\epsilon_{b, P}$, which we call the bias-corrected solution. In the examined cases the bias on $\phi_{P}$ is limited to a few percent, therefore, the bias-corrected solution resembles $\phi_{P}$.

\subsection{Discretization error}

The discretization error is typically assessed using Richardson extrapolation [12], where solutions on 3 successively finer grids are compared with each other. With a refinement 
factor of 2 , the discretization error on the finest grid $h$ is calculated as

$$
\epsilon_{d, h}=\frac{\phi_{h}-\phi_{2 h}}{2^{p}-1},
$$

where $\phi_{4 h}, \phi_{2 h}$ and $\phi_{h}$ are the solutions on the successively finer grids and $p$ the order of convergence, calculated as

$$
p=\frac{\log \left(\frac{\phi_{2 h}-\phi_{4 h}}{\phi_{h}-\phi_{2 h}}\right)}{\log (2)} .
$$

To obtain a reliable estimate of the discretization error, the statistical error and the bias should be sufficiently low.

Many factors can lead to an inaccurate estimate of the discretization error. To be able to have a well estimated order $p$, it is important that the grids are within the asymptotic range where $\epsilon_{d} \propto h^{p}$. Moreover, the presence of the sudden transition from the attached to the detached divertor regime can contribute to bad local estimates. When the estimate for $p$ is inaccurate, $p=1$ can be assumed in equation (7). Because of these inaccuracies, we take a safety factor of 1.25 into account. This is seen as good practice in CFD applications and recommended by Roache [13].

\section{Results}

In this section, we investigate the grid sensitivity of several variables. First, we examine the outer target profiles of the plasma heat flux and the electron temperature. Additionally, we evaluate whether the grids are sufficiently fine to resolve the gradients correctly. Afterwards, a density scan is performed by simulating different amounts of gas puff in order to analyze the operating space of the divertor and to determine the $I_{\text {sat }}$ roll-over point.

\subsection{Target profiles}

Figures 1 and 2 show the result of each simulation for the heat flux $q_{p}$ (equation (1)) and the electron temperature $T_{e}$ as function of distance $s$ along the outer divertor, with $s=0$ at the separatrix strike-point. The simulations differ in the grid size and number of MC particles. All the boundary conditions are kept the same. Results from the $74 \times 28$, 148x56 and 296x112 grids are indicated in respectively red, blue and black. For each grid, the results from the simulation with 700 and $7000 \mathrm{MC}$ particles per iteration are shown with respectively dashed and solid line.

A substantial change with grid refinement is the increase in the maximal heat flux. On the coarse grid, the maximum heat flux is approximately $2.3 \mathrm{MW} / \mathrm{m}^{2}$, whereas on the finest grid, its value is more than $40 \%$ higher: $3.3 \mathrm{MW} / \mathrm{m}^{2}$, from the most accurate solution with $P=7,000$. We also observe that the location of this maximum shifts more towards the separatrix with finer grids. A summary of the error contributions to the maximum heat flux for each simulation is given in table 1 . The statistical error is at most a few percent of each solution, because the solution is averaged over a large number 


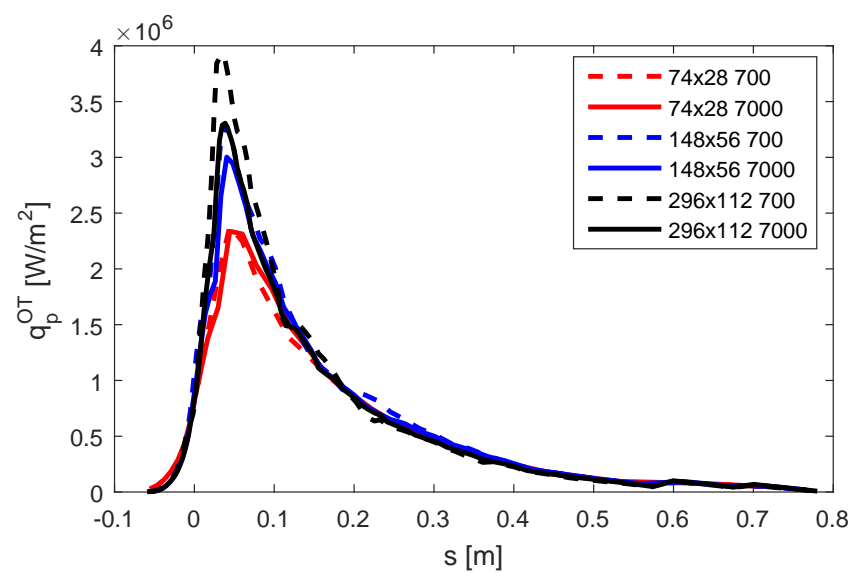

Figure 1: Heat flux density $q_{p}$ along the outer target for different grid sizes $(74 \mathrm{x} 28$, $148 \times 56,296 \times 112)$ and number of MC particles per iteration $(700,7000)$.

of iterations. The bias clearly increases with the grid size, which is expected because the variance of the results of each iteration increases with the grid size. For $P=7,000$, however, the bias is limited to a few percent, even on the finest grid. Finally, we observe that the discretization error is large. On the coarse grid, it is estimated to be $63 \%$, while on the finest grid, it is still approximately 11\%. Typically, the peak values are very sensitive to the cell size. In finite volume codes with first-order upwind discretization schemes, numerical or artificial diffusion is introduced which smears out the peaks and rapid variations. The rate of error reduction of this numerical diffusion is only first order; therefore, very fine grids are usually required to obtain accurate solutions [14].

\begin{tabular}{|c|c|c|c|c|c|c|}
\hline & $74 \times 28700$ & $74 \times 287000$ & $148 \times 56700$ & $148 \times 567000$ & $296 \times 112700$ & $296 \times 1127000$ \\
\hline$\epsilon_{s}$ & 0.6 & 0.2 & 0.8 & 0.2 & 2.3 & 0.3 \\
\hline$\epsilon_{b}$ & 4.2 & 0.4 & 19 & 2.1 & 35 & 4.2 \\
\hline$\epsilon_{d}$ & \multicolumn{2}{|c|}{63} & \multicolumn{2}{|c|}{23} & \multicolumn{2}{|c|}{11} \\
\hline
\end{tabular}

Table 1: Estimates for the relative error on the peak plasma heat flux $q_{p}[\%]$ for each simulation.

Increasing the number of the grid cells clearly steepens the electron temperature profile, as shown in figure 2. This is expected because similarly to the peak values, sharp transitions are typically sensitive to the grid resolution due to the increased impact of numerical diffusion on coarser grids. The profiles appear also to be very sensitive to the statistical noise. However, this fluctuating behavior cannot be explained by the statistical error and bias, but is believed to be due to the method of refining the grid. Each coarse grid cell is simply split into 4 or 16 to obtain the finer grids, without adapting for alignment with the magnetic field or assuring gradual changes in cell size. It is expected that the profiles become smoother when alignment is attained and no abrupt changes in cell size occur. 


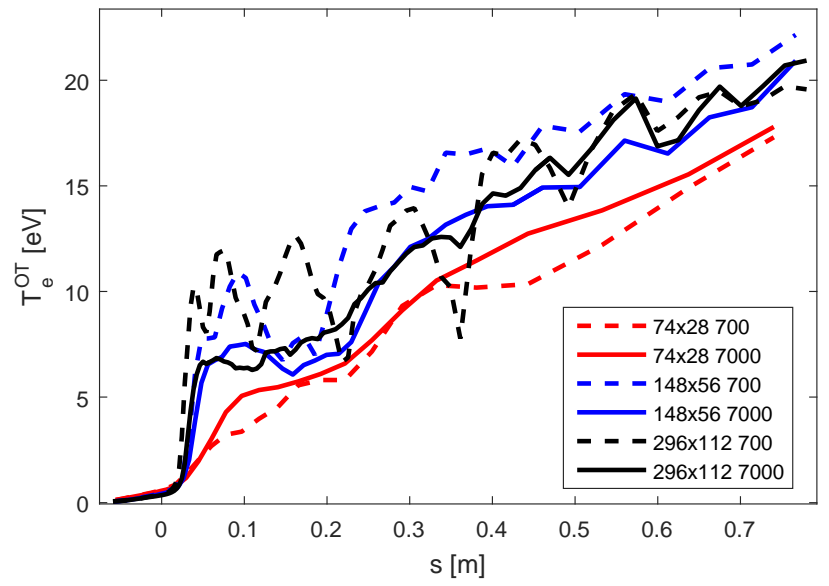

Figure 2: Electron temperature $T_{e}$ along the outer target for different grid sizes $(74 \times 28$, 148x56, 296x112) and number of MC particles per iteration (700, 7000).
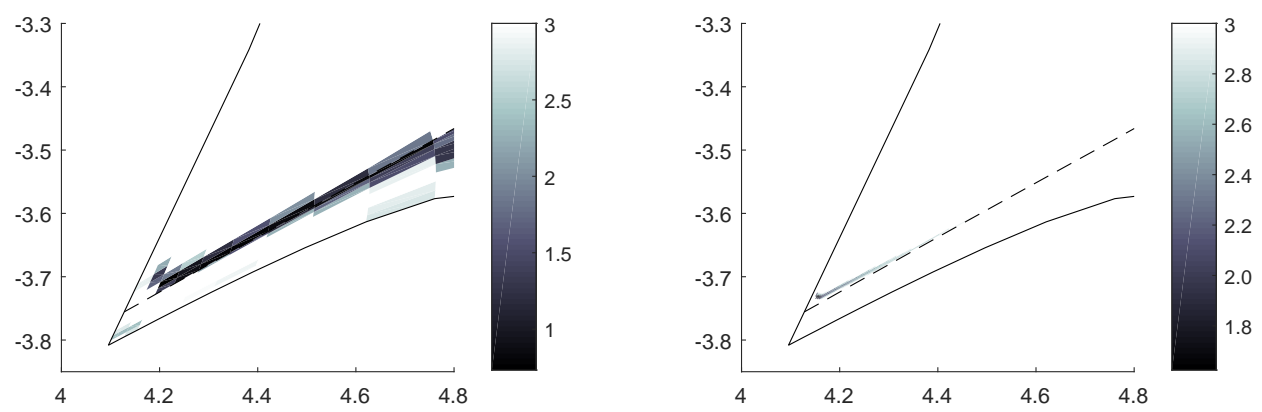

Figure 3: The length scale normalized to cell width $\frac{\lambda_{\phi}}{\Delta x}$ for values smaller than 3 for $T_{e}$ in the radial direction for the $74 \times 28$ grid (left) and the 296x112 grid (right) near the inner target. The full and dashed black lines indicate respectively the boundaries of the domain and the separatrix.
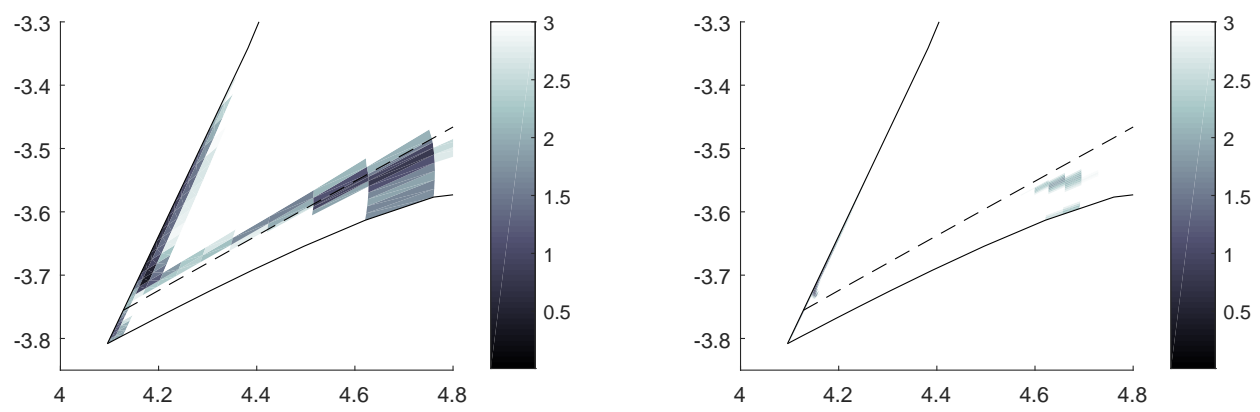

Figure 4: The length scale normalized to cell width $\frac{\lambda_{\phi}}{\Delta x}$ for values smaller than 3 for $T_{e}$ in the poloidal direction for the 74x28 grid (left) and the 296x112 grid (right) near the inner target. The full and dashed black lines indicate respectively the boundaries of the domain and the separatrix. 
To determine whether the grid resolution is adequate to resolve the gradients, the physical length scales $\lambda_{\phi}=\left|\frac{\phi}{\nabla \phi}\right|$ are compared with the cell width $\Delta x$ in the same direction (e.g. poloidal or radial). When at least 3 cells are present for each length scale, $\frac{\lambda_{\phi}}{\Delta x} \geq 3$, the grid is considered to be sufficiently fine to resolve the gradients correctly. Figures 3 and 4 show the values of $\frac{\lambda_{\phi}}{\Delta x}$ which are smaller than 3 for the electron temperature $T_{e}$ near the inner target for the coarsest and finest grid in respectively the radial and poloidal direction. The most sensitive regions are located near the separatrix in the divertor region and in front of the target. While large areas are underresolved with the coarse grid, only very small areas remain insufficiently resolved in the finest grid. Hardly visible in the figure, but still present in the fine grid is the first cell next to the target in the poloidal direction. Due to large amount of volume recombination in the first cells, a sudden jump occurs in the plasma variables to satisfy the Bohm criterion at the boundary. Even higher resolution in these underresolved areas is recommended to achieve more accurate solutions. Overall, however, the finest grid is expected to have discretization errors limited to $10-15 \%$.

\subsection{Density scan}

In systematic studies of the operational space of the ITER divertor, a density scan is often performed [4]. Three key quantities, namely the peak power at the target $q_{p k}$, the ion saturation current at the target $I_{\text {sat }}$ and the average neutral pressure $p_{D T}$ in the private flux region are then monitored to assess divertor operation. The peak power at the target $q_{p k}$ takes into account the plasma heat flux as discussed in the previous section (equation (1)), as well as the potential energy of the recombination of atoms to molecules for the molecular flux originated from ions and atoms and the kinetic energy taken away by the reflected neutrals. It also includes the power load from neutrals and radiation. The ion saturation current $I_{s a t}$ is calculated as the integral of the ion saturation current over the whole target section. The neutral pressure $p_{D T}$ includes the pressure of atoms and molecules. It is calculated as the sum of all contributions at the interface between the plasma and the private flux region. All these variables have been calculated with additional MC runs with a large number of MC particles in post-processing. The statistical error on each value is smaller than $1 \%$.

A series of simulations with decreasing strength of gas puff has been performed on the coarse $74 \times 28$ grid. This operating line for the outer target peak heat flux and the neutral pressure is represented by the full black line with dots in figure 5 (left). It reveals the decrease in the ion saturation current and peak heat power load with an increase of the neutral density/pressure near the pump as expected from the partly detached divertor behavior. Figure 5 (right) shows the decrease in the ion saturation current on the inner target with increasing neutral pressure. For the case \#2011, we summarize the deterministic error contributions for $p_{D T}, q_{p k}^{O T}$ and $I_{s a t}^{I T}$ in table 2. The errors are graphically shown in figure 5. For each grid size (diamonds for the intermediate grid size and crosses for the fine grid), the results of the simulation with 700 and 7,000 MC 
particles are indicated in respectively blue and red. Using the expected error reduction rate, the bias is estimated and the bias-corrected solution is extrapolated, indicated with the black symbol. The black dashed line connects the bias-corrected solutions for the three grid sizes. The difference between the bias-corrected solution and the obtained solution is the measure for the bias. By comparing the bias-corrected solutions on different grids, the discretization error is estimated. The discretization error for the peak power $q_{p k}^{O T}$ on the coarse grid is $35 \%$, which is substantially lower than the $63 \%$ when only the plasma power is taken into account (see table 1). The neutral pressure $p_{D T}$ and the ion saturation current $I_{\text {sat }}^{I T}$ have an even lower discretization error, because they are integral quantities, which are typically less sensitive to the grid size. On the fine grid, the discretization error of these variables is estimated to be $5-7 \%$, whereas the bias is $3-5 \%$.

\begin{tabular}{c|cc|cc} 
& \multicolumn{2}{|c}{$74 \times 28$} & 7000 & \multicolumn{2}{c}{$298 \times 1127000$} \\
& $\epsilon_{d}$ & $\epsilon_{b}$ & $\epsilon_{d}$ & $\epsilon_{b}$ \\
\hline$p_{D T}$ & 23 & 1 & 6 & 5 \\
$q_{p k}^{O T}$ & 35 & 0.1 & 7 & 5 \\
$I_{s a t}^{I T}$ & 16 & 0.04 & 5 & 3 \\
\hline
\end{tabular}

Table 2: Estimates for the relative error for $p_{D T}, q_{p k}^{O T}$ and $I_{s a t}^{I T}[\%]$.

To study the effect of the discretization on the whole operating line, a fine grid simulation (296x112) with 7,000 MC particles per iteration is performed for each case. The results for the inner and outer targets are shown with the red line in figure 6 and 7 , respectively. The results on the coarse $74 \times 28$ grid are indicated in black. One can see that the whole operating line shifts to higher $q_{p k}$ and lower $p_{D T}$. Comparing for the same model input, say, $p_{D T} \approx 6 \mathrm{~Pa}$, one would conclude that $q_{p k}^{I T}$ and $q_{p k}^{O T}$ are respectively 24 and $27 \%$ higher on the finer grid. However, it is more representative physically to compare the runs that are in a similar state with respect to plasma detachment, that is, to the $I_{\text {sat }}$ roll-over [1]. This way, one should compare the leftmost point $(2.5 \mathrm{~Pa})$ on the black line with the central point $(3.7 \mathrm{~Pa})$ on the red one. Looking this way, we see that whereas $q_{p k}$ on the inner divertor target is higher by $\approx 15 \%$ on the finer grid, on the outer, more loaded target it stays the same. The $q_{p k}$ increase resulting from the higher grid resolution is offset by operational window shifting towards higher $p_{D T}$ (see also ref. [1]). To properly evaluate the practical implications and to determine whether the shift in operational space interferes with the boundaries for the operating domain, a similar study should be performed for full burning plasma conditions.

It is noted that with the finest grid, a smaller time step $\left(\Delta t=2 \cdot 10^{-7} \mathrm{~s}\right)$ was necessary for convergence in the runs with higher gas puff. Numerical instabilities appeared near the separatrix in front of the inner target. The lack of convergence could also be detected from the value of the residuals of the average value. The blue line in figure 8 shows the residuals of the average with $\Delta t=10^{-6} \mathrm{~s}$ (all simulations used $P=7,000)$. Clearly, this residual starts to increase when the gas puff is larger than 
Grid resolution study for B2-EIRENE simulation of partially detached ITER divertor plasma11
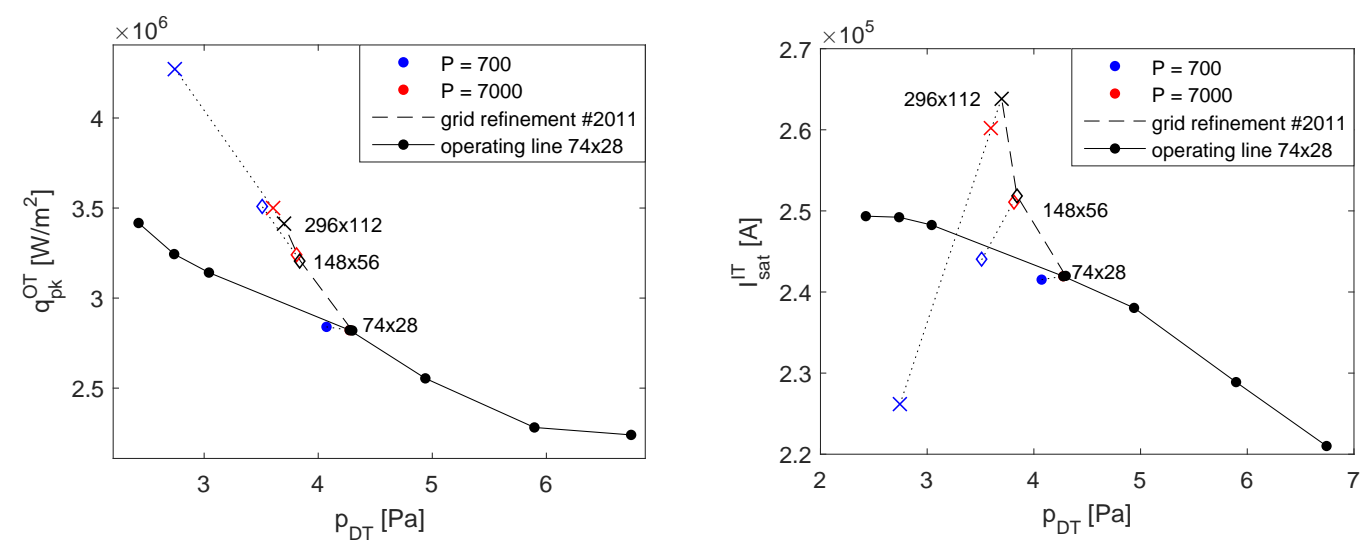

Figure 5: Variation of the peak power on the outer target $q_{p k}^{O T}$ (left) and the saturation current on the inner target $I_{\text {sat }}^{I T}$ (right) with the neutral pressure in the private flux region $p_{D T}$. Results indicated with circles, diamonds and crosses are obtained on the $74 \times 28$, $148 \times 56$ and 296x112 grid, respectively. The blue and red symbols are obtained with $P=700$ and $P=7,000$, respectively.
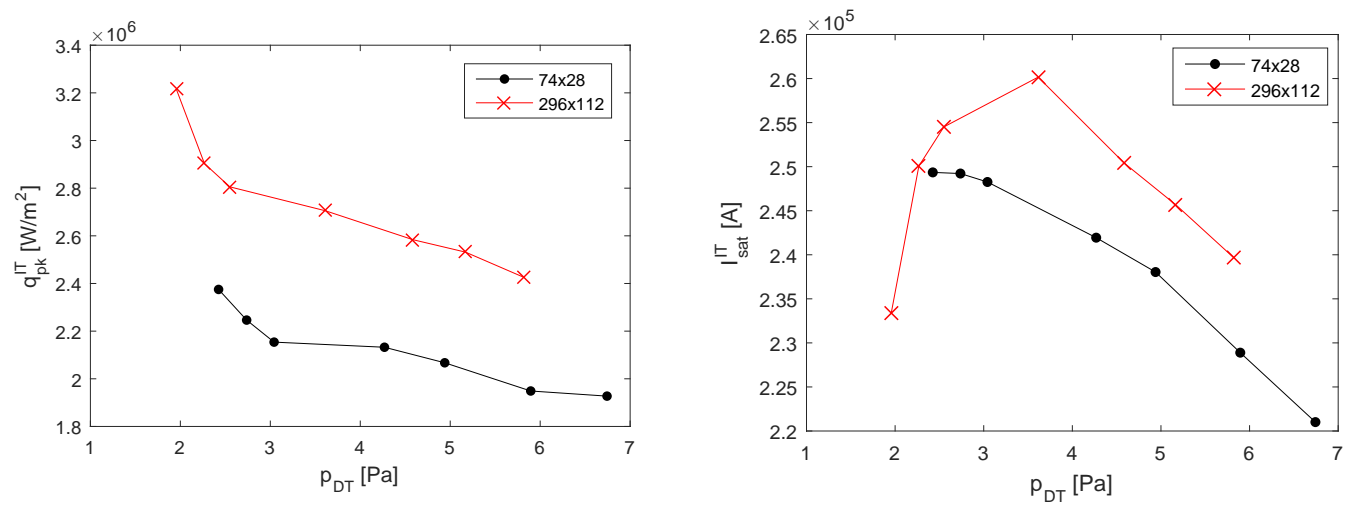

Figure 6: Operating line $q_{p k}$ (left) and $I_{s a t}$ (right) vs $p_{D T}$ on the coarse (black circles) and fine (red crosses) grid on the inner target.
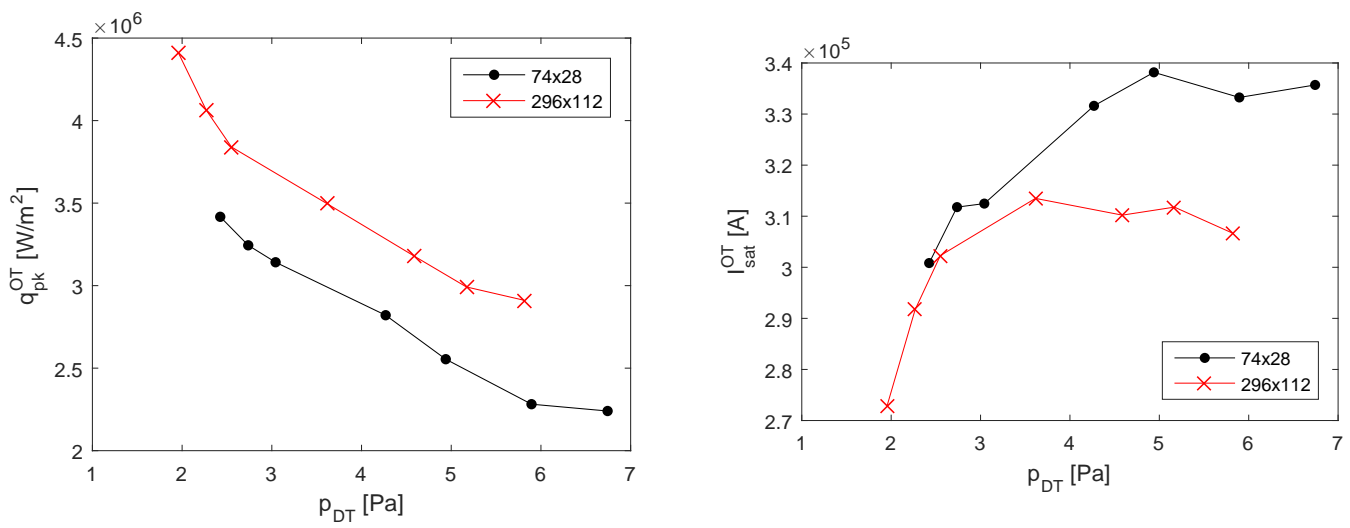

Figure 7: Operating line $q_{p k}$ (left) and $I_{s a t}$ (right) vs $p_{D T}$ on the coarse (black circles) and fine (red crosses) grid on the outer target. 
Grid resolution study for B2-EIRENE simulation of partially detached ITER divertor plasma12

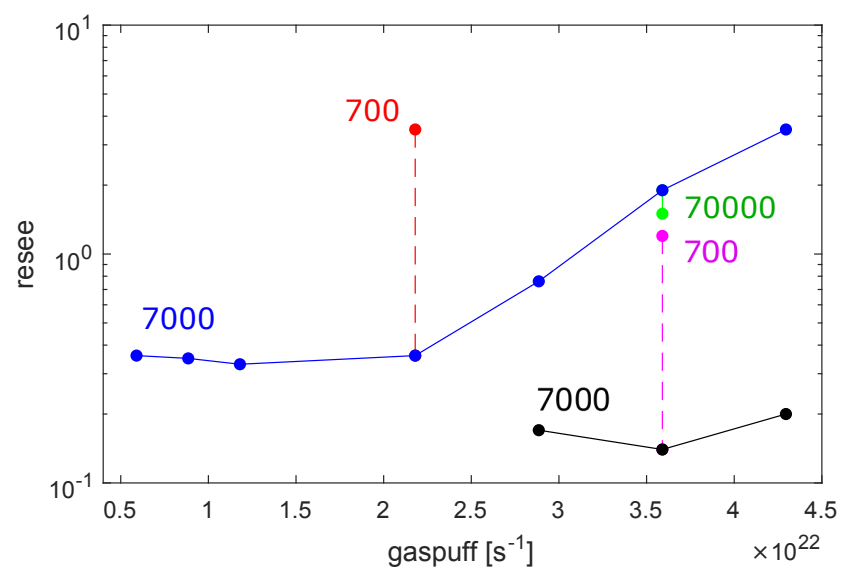

Figure 8: Residuals: blue line for $\Delta t=10^{-6} \mathrm{~s}$ (red circle with $P=700$, blue circles with $P=7,000$ and green circle with $P=70,000$ ); black line with $\Delta t=2 \cdot 10^{-7} \mathrm{~s}$ (pink circle with $P=700$, and black circles with $P=7,000)$

$\approx 2.5 \cdot 10^{22}$. Even with a larger number of MC particles per iteration (green point with $P=70,000)$, the residual does not decrease. With converged runs, the residual should decrease according to $R(\bar{\phi}) \propto \frac{1}{P}$, as can be seen from comparing the $P=7,000$ (blue) and $P=700$ (red) residual for \# 2011 (with a gas puff of $2.1810^{22} \mathrm{~s}^{-1}$ ). With a smaller time step $\left(\Delta t=2 \cdot 10^{-7} \mathrm{~s}\right)$, the residuals are lower and remain approximately constant with varying gas puff (black line in figure 8). Additionaly, the expected $\propto \frac{1}{P}$ scaling is obtained as can be seen by comparing the residuals of the $P=7,000$ runs in black with the residual of a $P=700$ run indicated in pink.

\section{Conclusion}

We examined the grid sensitivity in a B2-EIRENE simulation of a partially detached ITER divertor plasma. Because of the speed up gained by averaging, it was feasible to execute simulations on finer discretization grids, created by refining the original $74 \times 28$ grid (F12). A detailed analysis of the numerical errors has been carried out to evaluate the influence of the grid size and the limited number of MC particles. For the examined runs, it has been demonstrated that grid resolution has a large impact on the solution. On the original $74 \times 28$ grid, the discretization errors for the peak values can reach $30-60 \%$. The integral values are less sensitive with estimated discretization errors around $20 \%$. With higher grid resolution, the operating line of the divertor shifts to higher peak powers and roll-over occurs at a higher neutral pressure, and this offsets the effect of the increase of the peak power loading onto the targets. On the finest grid 296x112, we achieved a total numerical error of only 10-15\% on all examined variables. The grid sensitivity for cases with impurities at high power in the new F57 ITER divertor geometry [4] remains to be examined. 
Grid resolution study for B2-EIRENE simulation of partially detached ITER divertor plasma13

\section{Acknowledgments}

This work has been carried out within the framework of the EUROfusion Consortium and has received funding from the Euratom research and training programme 2014-2018 under grant agreement No.633053. The views and opinions expressed herein do not necessarily reflect those of the European Commission. This work is sponsored by Flanders Innovation and Entrepreneurship (IWT.141064).

\section{References}

[1] A. S. Kukushkin, H. D. Pacher, V. Kotov, G. W. Pacher, and D. Reiter, "Finalizing the ITER divertor design: The key role of SOLPS modeling," Fusion Engineering and Design, vol. 86, pp. 2865-2873, 2011.

[2] D. Reiter, M. Baelmans, and P. Börner, "The EIRENE and B2-EIRENE codes," Fusion Science and Technology, vol. 47, pp. 172-186, 2005.

[3] S. Wiesen, D. Reiter, V. Kotov, M. Baelmans, W. Dekeyser, A. Kukushkin, S. Lisgo, R. Pitts, V. Rozhansky, G. Saibene, I. Veselova, and S. Voskoboynikov, "The new SOLPS-ITER code package," Journal of Nuclear Materials, vol. 463, pp. 480 - 484, 2015.

[4] A. S. Kukushkin, H. D. Pacher, A. Loarte, V. Kotov, M. Merola, G. W. Pacher, and D. Reiter, "Analysis of performance of the optimized divertor in iter," Nuclear Fusion, vol. 49, p. 075008, 2009.

[5] K. Ghoos, W. Dekeyser, G. Samaey, and P. B "Accuracy and convergence of coupled finitevolume / monte-carlo codes for plasma edge simulations of nuclear fusion reactors," Journal of Computational Physics, vol. 322, pp. 162-182, 2016.

[6] M. Baelmans, P. Borner, K. Ghoos, and G. Samaey, "Efficient code simulation strategies for B2-EIRENE," Nuclear Materials and Energy, vol. 12, pp. 858-863, 2017.

[7] Y. Marandet, H. Bufferand, G. Ciraolo, P. Genesio, P. Meliga, J. Rosato, E. Serre, and P. Tamain, "Effect of statistical noise on simulation results with a plasma fluid code coupled to a monte carlo kinetic neutral code," Contributions to Plasma Physics, vol. 56, pp. 604-609, 2016.

[8] A. S. Kukushkin, H. D. Pacher, V. Kotov, D. Reiter, D. P. Coster, and G. W. Pacher, "Effect of the dome on divertor performance in ITER," Journal of Nuclear Materials, vol. 363-365, pp. 308-313, 2007.

[9] R. D. Smirnov, A. S. Kukushkin, S. I. Krasheninnikov, A. Y. Pigarov, and T. D. Rognlien, "Impurity-induced divertor plasma oscillations," Physics of Plasmas, vol. 23, no. 1, p. 012503, 2016.

[10] K. Ghoos, G. Samaey, and M. Baelmans, "Numerical error estimation in random noise coupled plasma edge simulations in nuclear fusion reactors," in Proc. of International Conference on Mathematics and Computational Methods Applied to Nuclear Science and Engineering, Jeju, Korea, 2017.

[11] J. Fan and Q. Yao, Nonlinear Time Series: Nonparametric and Parametric Methods. Springer Series in Statistics, 2003.

[12] P. J. Roache, "Perspective: A method for uniform reporting of grid refinement studies," Journal of Fluids Engineering, vol. 116, pp. 405-413, 1994.

[13] P. J. Roache, "Conservatism of the grid convergence index in finite volume computations on steady-state fluid flow and heat transfer," Journal of Fluids Engineering, vol. 125, pp. 731-732, 2003.

[14] J. H. Ferziger and M. Peric, Computational Methods for Fluid Dynamics. Berlin: Springer-Verlag, 2002. 